ABUNDANCE DETERMINATION IN WOLF-RAYET STARS

Anne B. Underhill

Department of Geophysics and Astronomy

University of British Columbia

Vancouver, B.C. V6T 1W5

\title{
1. PROPERTIES OF WOLF-RAYET STARS
}

Wolf-Rayet stars are rare and their basic properties such as mass, effective temperature, and luminosity are simflar to those of 09 to B1 stars. The spectra of Wolf-Rayet stars from about $10 \mu \mathrm{m}$ to $1000 \AA$ consist of rather broad emission lines on a continuous spectrum. The continuous spectrum corresponds to effective temperatures in the range from 25000 to $30000 \mathrm{~K}$ (Underhil1 1983b). A few shortward displaced absorption troughs appear and a few emission lines are flat topped. There is a strong infrared excess attributed to thermal bremsstrahlung (Cohen, Barlow, and Kuhi 1975), and there is rather strong $6 \mathrm{~cm}$ radiation (a fraction of a mJy from stars at distances of the order of $1500 \mathrm{pc}$ ), see for instance, Bieging, Abbott, and Churchwell (1982). The radio radiation can be attributed to thermal bremsstrahlung and to magnetic bremsstrahlung (cyclotron radiation), see Underhil1 (1983a). $X$ rays have been measured from a few Wolf-Rayet stars (Sanders, et al. 1985).

Wolf-Rayet stars show spectroscopic evidence for a wind, the maximum outflow velocity being of the order of 1500 to $2000 \mathrm{~km} \mathrm{~s}^{-1}$ (Underhill 1983a). The density of the wind is uncertain. One obtains an upper 1 imit of about $5 \times 10^{-5} \mathrm{M}_{\ominus} \mathrm{yr}^{-1}$ if one interprets the radio flux as due solely to thermal bremsstrahlung. Allowance for possible magnetic bremsstrahlung allows lower values.

The line emitting region appears to be very extensive (Bhatia and Underhill 1986). The photospheres of Wolf-Rayet stars are normal for Population I stars of their mass. In the mantles of WC stars $\mathrm{T}_{\mathrm{e}-3}<10^{5}$ $\mathrm{K}$ and $10^{9}<\mathrm{N}_{\mathrm{e}}<10^{10} \mathrm{~cm}^{3}$ while $\mathrm{T}_{\mathrm{e}}>10^{5} \mathrm{~K}$ and $10^{9}<\mathrm{N}_{\mathrm{e}}<10^{10} \mathrm{~cm}$ in the mantles of WN stars. These results come from Bhatia and Underhi11(1986) and from ongoing research. The composition of the atmosphere appears to be normal, that is solar.

\section{THEORETICAL MODELS}

Wolf-Rayet atmospheres are best modeled in terms of a photosphere surrounded by a mantle. Bhatia and Underhill (1986) have studied the 
$\mathrm{H}$ and He problem anew using realistic model atoms in the solution for statistical equilibrium. We are working on $\mathrm{C}, \mathrm{N}$, and 0 . We use the "one-representative-point" radiative transfer theory of Castor and van Blerkom (1970) and we use the best available expressions for the relevant radiative and collision cross sections. Our model atoms are as follows: $H$ has 17 levels, all carried in NLTE; He has 70 levels,all carried in NLTE; C has 139 levels, 44 carried in NLTE, 95 in LTE; $N$ has 139 levels, 38 carried in NLTE, 101 in LTE; 0 has 139 levels, 44 carried in NLTE, 95 in LTE. We have analysed the spectra of six WolfRayet stars.

\section{CONCLUSIONS}

From a study of $\mathrm{H}$, He I, He II, C II, C III, and C IV Iines we conclude: 1. The conspicuous patterns of relative line intensities in Wolf-Rayet spectra can be understood in terms of plasma with solar composition, high electron temperature $\left(\mathrm{T}_{\mathrm{e}^{\sim}} 10^{5} \mathrm{~K}\right)$, and moderate density $\left(10^{9}<\mathrm{N}_{\mathrm{e}}<\right.$ $10^{10} \mathrm{~cm}^{-3}$ ).

2. Much energy and momentum is injected into a very large mantle, possibly about 2500 times as much per unit volume as for the solar corona.

3. The extra energy and momentum may come from the differential rotation of the star. Reference to the Sun suggests that locally distributed, small magnetic fields play a key role in causing this energy and momentum transfer.

4. The winds from Wolf-Rayet stars probably have densities less than $10^{-6} \mathrm{M}_{0} \mathrm{yr}^{-1}$, thus the winds are of 1ittle influence on the evolution of the star.

5. The Wolf-Rayet stars are similar to $T$ Tauri stars in the sense that both groups require the action of an efficient energy and momentum transfer process in their mantles in order to generate line-emission flux of the order of 10 to $30 \%$ of the total stellar radiation field. 6. Wolf-Rayet stars are basically 09 to B1 stars. A key factor may be that the few stars known to be Wolf-Rayet stars were formed with larger than normal primordial magnetic fields.

7. The same energy/momentum mechanism probably works in some small, old stars, because some central stars of planetary nebulae show winds and some show Wolf-Rayet-type spectra.

8. A Wolf-Rayet spectrum is not a secure index of stage of evolution.

\section{REFERENCES}

Bhatia, A.K., and Underhil1, A.B. 1986 Ap.J. Suppl., in press. Castor, J.I., and van B1erkom, D. 1970, Ap.J., 161, 485. Cohen, M., Barlow, M.J., and Kuhi, L.V. 1975, Astr. Ap., 40, 291. Sanders, W.T. et. al., 1985, Ap.J., 288, 756. Und erhi11, A.B. 1983a, Ap.J., 265,933 . Underhill, A.B. 1983b, Ap.J., $2 \underline{2} 6$, 718 . 